\title{
Classifying finite-sheeted covering mappings of paracompact spaces
}

\author{
Vlasta MATIJEVIĆ \\ Department of Mathematics \\ University of Split \\ N. Tesle 12 \\ 21000 Split, Croatia \\ vlasta@pmfst.hr
}

Recibido: 11 de Octubre de 2001

Aceptado: 2 de Abril de 2002

\section{ABSTRACT}

The main result of the present paper is a classification theorem for finite-sheeted covering mappings over connected paracompact spaces. This theorem is a generalization of the classical classification theorem for covering mappings over a connected locally pathwise connected semi-locally 1-connected space in the finite-sheeted case. To achieve the result we use the classification theorem for overlay structures which was recently proved by S. Mardešić and V. Matijević (Theorems 1 and 4 of [5]).

2000 Mathematics Subject Classification: 57M10, 55P55, 55Q07, 54B35, 54C56.

Key words: Shape, resolution, inverse system, overlay. covering mapping, paracompact space, fundamental group, fundamental progroup.

\section{Introduction}

The well-known classical classification theorem of the covering space theory refers to covering mappings of connected spaces, where the base space is locally pathwise connected and semi-locally 1-connected. It establishes a bijection between classes of equivalent pointed $s$-sheeted covering mappings $f:(X, *) \rightarrow(Y, *)$ over $(Y, *)$ and subgroups $H$ of index $s$ of the fundamental group $G=\pi_{1}(Y, *)$. In the unpointed case, it establishes a bijection between classes of equivalent $s$-sheeted covering mappings $f: X \rightarrow Y$ over $Y$ and conjugacy classes of subgroups $H$ of index $s$ of the fundamental group $G=\pi_{1}(Y, *)$ (see, e.g., Theorems 8.1 and 8.4 of [1]).

In 1972, R.H. Fox, using the shape-theoretic approach, extended the classification theorem to arbitrary connected metric spaces. However, he had to restrict covering 
mappings to a narrower class, called overlay mappings. Fox has also noticed that for overlay mappings, connectedness of the total space $X$ has to be replaced by the indecomposability of the overlay mapping $f$, a property which he calls vertical connectedness of $f$. Moreover, he replaced $\pi_{1}(Y, *)$ by the appropriate shape-theoretic notion, i.e., by the fundamental progroup $\underline{\pi}_{1}(Y, *)$ (Theorem 6.1 of [2]). He considered only the unpointed case and instead of fundamental groups used the related notion of fundamental tropes. His classification theorem for $s$-sheeted overlay mappings over connected metric spaces was stated in terms of transitive representations of the fundamental trope in the symmetric group $\Sigma(s)$.

In 1978, T.T. Moore [7] introduced the notion of an overlay structure and exhibited examples of overlay mappings over connected metric spaces which admit different overlay structures. He also noticed that Fox has actually classified overlay structures over connected metric spaces and not overlay mappings.

Recently, S. Mardešić and V. Matijević classified indecomposable overlay structures over arbitrary connected topological spaces. Their classification theorem establishes a bijection between the set of all pointed equivalence classes of $s$-sheeted indecomposable overlay structures over connected topological spaces $(Y, *)$ and the set of all subprogroups of index $s$ of the fundamental progroup $\underline{\pi}_{1}(Y, *)$ (Theorem 1 of [5]). In the unpointed case, it establishes a bijection between the set of all equivalence classes of $s$-sheeted indecomposable overlay structures over connected topological spaces $Y$ and the set of all conjugacy classes of subprogroups of index $s$ of the fundamental progroup $\underline{\pi}_{1}(Y, *)$, where $*$ is an arbitrary point of $Y$ (Theorem 4 of [5]). These results are achieved using ANR-resolutions, an important technique introduced in [4], the fact that overlay mappings are pull-backs of covering mappings over ANR's (see [8]) and the classical classification theorem. Although these classification theorems are rather general, the problem of classifying covering mappings (in distiction to covering structures) over arbitrary connected topological spaces is still open.

The main result of the present paper is a classification theorem (see Theorems 4 and 5) for finite-sheeted covering mappings with connected total space over connected paracompact spaces. This theorem can be considered a proper generalization of the classical classification theorem of the covering space theory. It is actually a restriction of the Mardešić and Matijević classification theorem to the finite-sheeted case, because, in the finite-sheeted case, equivalence classes of covering mappings over a connected paracompact space $(Y, *)$ with a connected total space $(X, *)$ coincide with indecomposable overlay structures over $(Y, *)$ (Theorems 1,2 and 3 ).

\section{Overlay structures for finite-sheeted covering mappings of paracompact spaces}

By a paracompact space we shall mean Hausdorff paracompact space. Recall that the class of paracompact spaces contains the class of compact Hausdorff spaces and the 
class of metric spaces as its proper subclasses. Recall that each paracompact space is normal. Also recall that an open covering is called normal provided it admits a subordinated partition of unity. Since each locally finite open covering of a normal space is normal ([6], Appendix 1, 3.1 Theorem 1), in paracompact spaces all open coverings are normal.

Let $Y$ be a connected topological space, let $f: X \rightarrow Y$ be a continuous mapping and let $S$ be a set of cardinality $s=\operatorname{card} S$. Let $\mathcal{B}=\{B\}$ be an open covering of $Y$ and let $\mathcal{A}=\left\{A_{B}^{\sigma}: B \in \mathcal{B}, \sigma \in S\right\}$ be an open covering of $X$. We will say that $(\mathcal{A}, \mathcal{B})$ is an s-sheeted covering pair for $f: X \rightarrow Y$ provided the following three conditions are fulfilled:

(C1) $f^{-1}(B)=\bigcup_{\sigma \in S} A_{B}^{\sigma}, B \in \mathcal{B}$

(C2) $A_{B}^{\sigma} \cap A_{B}^{\tau}=\emptyset$, for $\sigma, \tau \in S, \sigma \neq \tau ; B \in \mathcal{B}$;

(C3) $\left.f\right|_{A_{B}^{\sigma}}: A_{B}^{\sigma} \rightarrow B$ is a homeomorphism for each $A_{B}^{\sigma} \in \mathcal{A}$.

Recall that a mapping $f: X \rightarrow Y$ is an $s$-sheeted covering mapping provided it admits an $s$-sheeted covering pair $(\mathcal{A}, \mathcal{B})$.

An $s$-sheeted covering pair $(\mathcal{A}, \mathcal{B})$ for $f: X \rightarrow Y$ is said to be an $s$-sheeted overlay pair for $f$ provided $\mathcal{B}$ is a normal covering and the following additional condition is fulfilled:

(C4) If $B, B^{\prime} \in \mathcal{B}$ and $B \cap B^{\prime} \neq \emptyset$, then every $\sigma \in S$ admits a unique $\sigma^{\prime} \in S$ such that $A_{B}^{\sigma} \cap A_{B^{\prime}}^{\sigma^{\prime}} \neq \emptyset$.

A mapping $f: X \rightarrow Y$ between topological spaces is said to be an s-sheeted overlay mapping provided it admits an $s$-sheeted overlay pair. Clearly, every overlay mapping is a covering mapping. In the realm of metric spaces the converse implication holds in some important cases. In particular, it holds if the base $Y$ is locally connected (see Theorem 3 in [3] or Proposition 2.1 in [7]) or if the number of sheets $s$ is finite (see Theorem 3 in [3] or Proposition 2.2 in [7]). Fox has given an example of a covering mapping over a metric continuum, which is not an overlay mapping (see [3]). Clearly, in that example the number of sheets is infinite.

If $(\mathcal{A}, \mathcal{B})$ and $\left(\mathcal{A}^{\prime}, \mathcal{B}^{\prime}\right)$ are $s$-sheeted covering (overlay) pairs for $f$, we say that the first pair refines the second one, and we write $(\mathcal{A}, \mathcal{B}) \leq\left(\mathcal{A}^{\prime}, \mathcal{B}^{\prime}\right)$, provided, for every $B \in \mathcal{B}$ there exists a $B^{\prime} \in \mathcal{B}^{\prime}$ such that $B \subseteq B^{\prime}$ and for every $\sigma \in S$, there exists a $\sigma^{\prime} \in S$ such that $A_{B}^{\sigma} \subseteq{A^{\prime}}_{B^{\prime}}^{\sigma^{\prime}}$. We say that two $s$-sheeted covering (overlay) pairs $(\mathcal{A}, \mathcal{B})$ and $\left(\mathcal{A}^{\prime}, \mathcal{B}^{\prime}\right)$ for $f$ are equivalent if there exists an $s$-sheeted covering (overlay) pair $\left(\mathcal{A}^{\prime \prime}, \mathcal{B}^{\prime \prime}\right)$ for $f$, which is their common refinement. By an $s$-sheeted covering structure (overlay structure) $[\mathcal{A}, \mathcal{B}]$ for a covering (overlay) mapping $f$ we mean an equivalence class of $s$-sheeted covering (overlay) pairs $(\mathcal{A}, \mathcal{B})$ for $f$. By an $s$-sheeted 
covering structure ( overlay structure) over a space $Y$, we mean an $s$-sheeted covering (overlay) structure for some mapping $f: X \rightarrow Y$.

Let $\mathcal{U}$ and $\mathcal{V}$ be coverings of a space $X$. If $\mathcal{U}$ refines $\mathcal{V}$, we shall write $\mathcal{U} \leq \mathcal{V}$.

Theorem 1. Let $f: X \rightarrow Y$ be an s-sheeted covering mapping over a connected paracompact space $Y$. If the number of sheets $s$ is finite, $f: X \rightarrow Y$ is an s-sheeted overlay mapping. Moreover, $f$ admits a unique s-sheeted overlay structure.

Proof. We need to show that $f: X \rightarrow Y$ admits an $s$-sheeted overlay pair $(\mathcal{A}, \mathcal{B})$ and that, for any two $s$-sheeted overlay pairs $\left(\mathcal{A}^{\prime}, \mathcal{B}^{\prime}\right),\left(\mathcal{A}^{\prime \prime} \mathcal{B}^{\prime \prime}\right)$ for $f$, there exists an $s$-sheeted overlay pair $\left(\mathcal{A}^{\prime \prime \prime}, \mathcal{B}^{\prime \prime \prime}\right)$, which is their common refinement. Since each $s$-sheeted overlay pair for $f$ is an $s$-sheeted covering pair for $f$, the theorem will be proved, if we prove the following claim.

Claim. If $\left(\mathcal{A}^{\prime}, \mathcal{B}^{\prime}\right)$ and $\left(\mathcal{A}^{\prime \prime} \mathcal{B}^{\prime \prime}\right)$ are $s$-sheeted covering pairs for $f$, then there is an $s$-sheeted overlay pair $(\mathcal{A}, \mathcal{B})$ for $f$, which is their common refinement.

Let us prove the claim. There is no loss of generality in assuming that $\mathcal{B}^{\prime}=\mathcal{B}^{\prime \prime}$. Indeed, if this is not the case, we can take a common refinement $\mathcal{B}^{\prime \prime \prime}$ of $\mathcal{B}^{\prime}$ and $\mathcal{B}^{\prime \prime}$ and the restrictions of $\left(\mathcal{A}^{\prime}, \mathcal{B}^{\prime}\right)$ and $\left(\mathcal{A}^{\prime \prime}, \mathcal{B}^{\prime \prime}\right)$ to $\mathcal{B}^{\prime \prime \prime}$. Let $\mathcal{A}^{\prime}=\left\{A_{B}^{\prime \sigma}: B \in \mathcal{B}^{\prime}, \sigma \in S\right\}$ and $\mathcal{A}^{\prime \prime}=\left\{A_{B}^{\prime \prime \sigma}: B \in \mathcal{B}^{\prime}, \sigma \in S\right\}$, card $S=s$. Since $Y$ is paracompact, $X$ is also a paracompact space (see [10]). Therefore, there exists an open covering $\mathcal{U}$ of $X$ such that $\mathrm{St}^{3} \mathcal{U} \leq \mathcal{A}^{\prime}, \mathcal{A}^{\prime \prime}$. For each point $y \in Y$ choose a member $B_{y} \in \mathcal{B}^{\prime}$ such that $y \in B_{y}$. Let $x$ be an arbitrary point from the fiber $f^{-1}(y)$. Then there exist a unique $\sigma \in S$ such that $x \in A_{B_{y}}^{\prime \sigma}$ and a unique $\tau \in S$ such that $x \in A_{B_{y}}^{\prime \prime \tau}$. Consequently, the set $S_{y}=\left\{(\sigma, \tau): f^{-1}(y) \cap A_{B_{y}}^{\prime \sigma} \cap A_{B_{y}}^{\prime \prime \tau} \neq \emptyset\right\} \subseteq S \times S$ has cardinality card $S_{y}=s$. Note that if $(\sigma, \tau),\left(\sigma^{\prime}, \tau^{\prime}\right) \in S_{y}$ and $(\sigma, \tau) \neq\left(\sigma^{\prime}, \tau^{\prime}\right)$ then $\sigma \neq \sigma^{\prime}$ and $\tau \neq \tau^{\prime}$. If $x=f^{-1}(y) \cap A_{B_{y}}^{\prime \sigma} \cap A_{B_{y}}^{\prime \prime \tau}$, let us denote $x$ by $x^{(\sigma, \tau)}$. For $(\sigma, \tau) \in S_{y}$ let $U_{y}^{(\sigma, \tau)}=$ $\operatorname{St}\left(x^{(\sigma, \tau)}, \mathcal{U}\right) \cap A_{B_{y}}^{\prime \sigma} \cap A_{B_{y}}^{\prime \prime \tau}, W_{y}=\bigcap_{(\sigma, \tau) \in S_{y}} f\left(U_{y}^{(\sigma, \tau)}\right)$ and $V_{y}^{(\sigma, \tau)}=f^{-1}\left(W_{y}\right) \cap A_{B_{y}}^{\prime \sigma} \cap A_{B_{y}}^{\prime \prime \tau}$. Since card $S_{y}=s$ is finite and $f$ is an open mapping, $W_{y}$ is an open set and, obviously, $y \in W_{y} \subseteq B_{y}$. Furthermore, $f^{-1}\left(W_{y}\right) \subseteq \bigcup_{(\sigma, \tau) \in S_{y}} U_{y}^{(\sigma, \tau)}$. Put $\mathcal{B}=\left\{W_{y}: y \in Y\right\}$. $\mathcal{B}$ is an open covering of $Y$, which refines $\mathcal{B}^{\prime}$. Since for each $y \in Y$, the cardinality $\operatorname{card} S_{y}=\operatorname{card} S=s$, there exists a bijection $\varphi_{y}: S \rightarrow S_{y}$. Put now $A_{W_{y}}^{\lambda}=V_{y}^{\varphi_{y}(\lambda)}$ and let $\mathcal{A}=\left\{A_{W_{y}}^{\lambda}: W_{y} \in \mathcal{B}, \lambda \in S\right\}$. $\mathcal{A}$ is an open covering of $X$, which refines both $\mathcal{A}^{\prime}$ and $\mathcal{A}^{\prime \prime}$. We claim that $(\mathcal{A}, \mathcal{B})$ is an $s$-sheeted overlay pair for $f$.

To prove $(\mathrm{C} 1)$ we need to show that $f^{-1}\left(W_{y}\right)=\bigcup_{\lambda \in S} A_{W_{y}}^{\lambda}=\bigcup_{\lambda \in S} V_{y}^{\varphi_{y}(\lambda)}=$ $\bigcup_{(\sigma, \tau) \in S_{y}} V_{y}^{(\sigma, \tau)}$, for each $W_{y} \in \mathcal{B}$. By definition of the sets $V_{y}^{(\sigma, \tau)}$, it is clear that $(\sigma, \tau) \in S_{y}$

$\bigcup_{(\sigma, \tau) \in S_{y}} V_{y}^{(\sigma, \tau)} \subseteq f^{-1}\left(W_{y}\right)$. So, we need to prove that $f^{-1}\left(W_{y}\right) \subseteq \bigcup_{(\sigma, \tau) \in S_{y}} V_{y}^{(\sigma, \tau)}$. 
Let $x^{\prime} \in f^{-1}\left(W_{y}\right)$. Then $f\left(x^{\prime}\right) \in W_{y}=\bigcap_{(\sigma, \tau) \in S_{y}} f\left(U_{y}^{(\sigma, \tau)}\right)$. Let $(\sigma, \tau) \in S_{y}$ be an arbitrary index. It follows that $f\left(x^{\prime}\right) \in f\left(U_{y}^{(\sigma, \tau)}\right) \subseteq f\left(A_{B_{y}}^{\prime \sigma} \cap A_{B_{y}}^{\prime \prime \tau}\right)$. Since

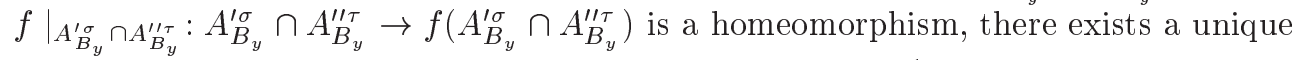
point $x^{\prime \prime} \in A_{B_{y}}^{\prime \sigma} \cap A_{B_{y}}^{\prime \prime \tau}$ such that $f\left(x^{\prime \prime}\right)=f\left(x^{\prime}\right)$, i.e. $x^{\prime \prime} \in f^{-1}\left(f\left(x^{\prime}\right)\right) \cap A_{B_{y}}^{\prime \sigma} \cap A_{B_{y}}^{\prime \prime \tau}$. So, for the choosen $(\sigma, \tau) \in S_{y}$ we can find a unique $x^{\prime \prime} \in f^{-1}\left(f\left(x^{\prime}\right)\right) \cap A_{B_{y}}^{\prime \sigma} \cap A_{B_{y}}^{\prime \prime \tau}$. Since card $S_{y}=s$ it follows that $f^{-1}\left(f\left(x^{\prime}\right)\right)=\bigcup_{(\sigma, \tau) \in S_{y}} f^{-1}\left(f\left(x^{\prime}\right)\right) \cap A_{B_{y}}^{\prime \sigma} \cap A_{B_{y}}^{\prime \prime \tau}$. Therefore, there must exist an index $\left(\sigma_{0}, \tau_{0}\right) \in S_{y}$ such that $x^{\prime}=f^{-1}\left(f\left(x^{\prime}\right)\right) \cap A_{B_{y}}^{\prime \sigma_{0}} \cap A_{B_{y}}^{\prime \prime \tau_{0}}$. Since $f\left(x^{\prime}\right) \in W_{y}$, it follows that $x^{\prime} \in f^{-1}\left(W_{y}\right) \cap A_{B_{y}}^{\prime \sigma_{0}} \cap A_{B_{y}}^{\prime \prime \tau_{0}}$. Now, we get $x^{\prime} \in V_{y}^{\left(\sigma_{0}, \tau_{0}\right)} \subseteq$ $\bigcup_{(\sigma, \tau) \in S_{y}} V_{y}^{(\sigma, \tau)}$, which proves $f^{-1}\left(W_{y}\right) \subseteq \bigcup_{(\sigma, \tau) \in S_{y}} V_{y}^{(\sigma, \tau)}$.

To prove (C2) we need to show that for different indices $\lambda, \lambda^{\prime} \in S$ the sets $A_{W_{y}}^{\lambda}=$ $V_{y}^{\varphi_{y}(\lambda)}=V_{y}^{(\sigma, \tau)}$ and $A_{W_{y}}^{\lambda^{\prime}}=V_{y}^{\varphi_{y}\left(\lambda^{\prime}\right)}=V_{y}^{\left(\sigma^{\prime}, \tau^{\prime}\right)}$ are disjoint. Since $\varphi_{y}: S \rightarrow S_{y}$ is a bijection and $\lambda \neq \lambda^{\prime}, \varphi_{y}(\lambda)=(\sigma, \tau)$ and $\varphi_{y}\left(\lambda^{\prime}\right)=\left(\sigma^{\prime}, \tau^{\prime}\right)$ are different elements of $S_{y}$. Then $\sigma \neq \sigma^{\prime}$ and $\tau \neq \tau^{\prime}$ and, therefore, $A_{B_{y}}^{\prime \sigma} \cap A_{B_{y}}^{\prime \sigma^{\prime}}=\emptyset$ and also $A_{B_{y}}^{\prime \prime \tau} \cap A_{B_{y}}^{\prime \prime \tau^{\prime}}=\emptyset$. Since $V_{y}^{(\sigma, \tau)} \cap V_{y}^{\left(\sigma^{\prime}, \tau^{\prime}\right)}=\left(f^{-1}\left(W_{y}\right) \cap A_{B_{y}}^{\prime \sigma} \cap A_{B_{y}}^{\prime \prime \tau}\right) \cap\left(f^{-1}\left(W_{y}\right) \cap A_{B_{y}}^{\prime \sigma} \cap A_{B_{y}}^{\prime \prime \tau^{\prime}}\right)=\emptyset$, it follows that $V_{y}^{(\sigma, \tau)}$ and $V_{y}^{\left(\sigma^{\prime}, \tau^{\prime}\right)}$ are disjoint.

To prove (C3) we need to show that for each $\lambda \in S,\left.f\right|_{A_{W_{y}}^{\lambda}}: A_{W_{y}}^{\lambda} \rightarrow W_{y}$ is a homeomorphism. Let $A_{W_{y}}^{\lambda}=V_{y}^{\varphi_{y}(\lambda)}=V_{y}^{(\sigma, \tau)}$. Since $V_{y}^{(\sigma, \tau)}=f^{-1}\left(W_{y}\right) \cap A_{B_{y}}^{\prime \sigma} \cap A_{B_{y}}^{\prime \prime \tau} \subseteq$

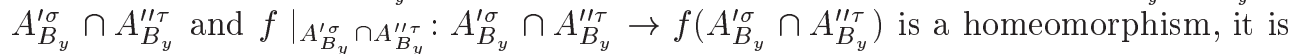
sufficient to prove that $f\left(V_{y}^{(\sigma, \tau)}\right)=W_{y}$. It is clear that $f\left(V_{y}^{(\sigma, \tau)}\right) \subseteq W_{y}$, so we need only to prove that $W_{y} \subseteq f\left(V_{y}^{(\sigma, \tau)}\right)$. Let $y^{\prime} \in W_{y}$. Since $W_{y}=\bigcap_{\left(\sigma^{\prime}, \tau^{\prime}\right) \in S_{y}} f\left(U_{y}^{\left(\sigma^{\prime}, \tau^{\prime}\right)}\right) \subseteq$ $f\left(U_{y}^{(\sigma, \tau)}\right)=f\left(\operatorname{St}\left(x^{(\sigma, \tau)}, \mathcal{U}\right) \cap A_{B_{y}}^{\prime \sigma} \cap A_{B_{y}}^{\prime \prime \tau}\right) \subseteq f\left(A_{B_{y}}^{\prime \sigma} \cap A_{B_{y}}^{\prime \prime \tau}\right)$, it follows that there exists a point $x^{\prime} \in A_{B_{y}}^{\prime \sigma} \cap A_{B_{y}}^{\prime \prime \tau}$ such that $f\left(x^{\prime}\right)=y^{\prime} \in W_{y}$. So, $x^{\prime} \in f^{-1}\left(W_{y}\right) \cap A_{B_{y}}^{\prime \sigma} \cap A_{B_{y}}^{\prime \prime \tau}=$ $V_{y}^{(\sigma, \tau)}$. Therefore, $y^{\prime}=f\left(x^{\prime}\right) \in f\left(V_{y}^{(\sigma, \tau)}\right)$.

(C4) Let $W_{y_{1}} \cap W_{y_{2}} \neq \emptyset$. We need to prove that for each $\lambda \in S$ there exists a unique $\lambda^{\prime} \in S$ such that $A_{W_{y_{1}}}^{\lambda} \cap A_{W_{y_{2}}}^{\lambda^{\prime}} \neq \emptyset$. Assume the contrary. Let $\lambda^{\prime \prime} \in S$ be another index, $\lambda^{\prime \prime} \neq \lambda^{\prime}$, such that $A_{W_{y_{1}}}^{\lambda} \cap A_{W_{y_{2}}}^{\lambda^{\prime \prime}} \neq \emptyset$. Let $A_{W_{y_{1}}}^{\lambda}=V_{y_{1}}^{\varphi_{y_{1}}(\lambda)}=V_{y_{1}}^{(\sigma, \tau)}, A_{W_{y_{2}}}^{\lambda^{\prime}}=$ $V_{y_{2}}^{\varphi_{y_{2}}\left(\lambda^{\prime}\right)}=V_{y_{2}}^{\left(\sigma^{\prime}, \tau^{\prime}\right)}, A_{W_{y_{2}}}^{\lambda^{\prime \prime}}=V_{y_{2}}^{\varphi_{y_{2}}\left(\lambda^{\prime \prime}\right)}=V_{y_{2}}^{\left(\sigma^{\prime \prime}, \tau^{\prime \prime}\right)}$. Since $\varphi_{y_{2}}: S \rightarrow S_{y_{2}}$ is a bijection and $\lambda^{\prime} \neq \lambda^{\prime \prime}, \varphi_{y_{2}}\left(\lambda^{\prime}\right)=\left(\sigma^{\prime}, \tau^{\prime}\right)$ and $\varphi_{y_{2}}\left(\lambda^{\prime \prime}\right)=\left(\sigma^{\prime \prime}, \tau^{\prime \prime}\right)$ are different elements of the set $S_{y_{2}}$. Then $x_{2}^{\left(\sigma^{\prime}, \tau^{\prime}\right)} \neq x_{2}^{\left(\sigma^{\prime \prime}, \tau^{\prime \prime}\right)}$, where $x_{2}^{\left(\sigma^{\prime}, \tau^{\prime}\right)}=f^{-1}\left(y_{2}\right) \cap A_{B_{y_{2}}}^{\prime \sigma^{\prime}} \cap A_{B_{y_{2}}}^{\prime \prime \tau^{\prime}}$ and $x_{2}^{\left(\sigma^{\prime \prime}, \tau^{\prime \prime}\right)}=$ $f^{-1}\left(y_{2}\right) \cap A_{B_{y_{2}}}^{\prime \sigma^{\prime \prime}} \cap A_{B_{y_{2}}}^{\prime \prime \tau^{\prime \prime}}$. Since $V_{y_{1}}^{(\sigma, \tau)} \subseteq \operatorname{St}\left(x_{1}^{(\sigma, \tau)}, \mathcal{U}\right) \cap A_{B_{y_{1}}}^{\prime \sigma} \cap A_{B_{y_{1}}}^{\prime \prime \tau} \subseteq \operatorname{St}\left(x_{1}^{(\sigma, \tau)}, \mathcal{U}\right)$ and analogously $V_{y_{2}}^{\left(\sigma^{\prime}, \tau^{\prime}\right)} \subseteq \operatorname{St}\left(x_{2}^{\left(\sigma^{\prime}, \tau^{\prime}\right)}, \mathcal{U}\right), V_{y_{2}}^{\left(\sigma^{\prime \prime}, \tau^{\prime \prime}\right)} \subseteq \operatorname{St}\left(x_{2}^{\left(\sigma^{\prime \prime}, \tau^{\prime \prime}\right)}, \mathcal{U}\right)$, it follows by as- 
sumption that $\operatorname{St}\left(x_{1}^{(\sigma, \tau)}, \mathcal{U}\right) \cap \operatorname{St}\left(x_{2}^{\left(\sigma^{\prime}, \tau^{\prime}\right)}, \mathcal{U}\right) \neq \emptyset$ and $\operatorname{St}\left(x_{1}^{(\sigma, \tau)}, \mathcal{U}\right) \cap \operatorname{St}\left(x_{2}^{\left(\sigma^{\prime \prime}, \tau^{\prime \prime}\right)}, \mathcal{U}\right) \neq \emptyset$.

Therefore $\quad x_{2}^{\left(\sigma^{\prime}, \tau^{\prime}\right)}, x_{2}^{\left(\sigma^{\prime \prime}, \tau^{\prime \prime}\right)} \in \quad \operatorname{St}\left(\operatorname{St}\left(x_{1}^{(\sigma, \tau)}, \mathcal{U}\right), \operatorname{St} \mathcal{U}\right) \quad \subseteq$ $\operatorname{St}\left(x_{1}^{(\sigma, \tau)}, \mathrm{St}^{2} \mathcal{U}\right)$. Since $\mathrm{St}^{3} \mathcal{U} \leq \mathcal{A}^{\prime}, \mathcal{A}^{\prime \prime}$ there exist $B \in \mathcal{B}^{\prime}$ and $\sigma \in S$ such that $x_{2}^{\left(\sigma^{\prime}, \tau^{\prime}\right)}, x_{2}^{\left(\sigma^{\prime \prime}, \tau^{\prime \prime}\right)} \in A_{B}^{\sigma \sigma}$. But, this contradicts the fact that $\left.f\right|_{A_{B}^{\prime \sigma}}: A_{B}^{\prime \sigma} \rightarrow B$ is a homeomorphism since $f\left(x_{2}^{\left(\sigma^{\prime}, \tau^{\prime}\right)}\right)=f\left(x_{2}^{\left(\sigma^{\prime \prime}, \tau^{\prime \prime}\right)}\right)=y_{2}$. So, we have proved (C4).

Since, $(\mathcal{A}, \mathcal{B})$ is the $s$-sheeted overlay pair and $(\mathcal{A B}) \leq\left(\mathcal{A}^{\prime}, \mathcal{B}^{\prime}\right),\left(\mathcal{A}^{\prime \prime} \mathcal{B}^{\prime}\right)$ the claim is proved.

\section{Indecomposable finite-sheeted overlay structures over paracompact spaces}

In classical covering space theory one always requires that the total space $X$ be connected. Therefore we shall now consider finite-sheeted covering mappings $f$ : $X \rightarrow Y$ with connected total space over a connected paracompact space. According to Theorem 1, it is sufficient to consider finite-sheeted overlay mappings $f: X \rightarrow Y$ with connected total space over a connected paracompact space, because in the finitesheeted case the notions of an overlay mapping and of a covering mapping coincide. As we mentioned before, Fox noticed that for overlay mappings, connectedness of the total space $X$ has to be replaced by some property of the overlay mapping $f$, which we call the indecomposability of $f$. Our aim in this section is to prove that in the finite-sheeted case connectedness of the total space and indecomposability coincide (see [5]).

Let us first recall the definition of a decomposable overlay structure (see [5]). We say that an overlay pair $(\mathcal{A}, \mathcal{B})$ for a mapping $f: X \rightarrow Y$ is decomposable provided there exist non-empty disjoint open sets $X^{1}, X^{2}$, whose union is $X$, and there exist non-empty disjoint subsets $S^{1}, S^{2}$, whose union is $S$. Moreover, the collections $\mathcal{A}^{i}=$ $\left(A_{B}^{\sigma^{i}}, B \in \mathcal{B}, \sigma^{i} \in S^{i}\right), i=1,2$, together with $\mathcal{B}$ form overlay pairs $\left(\mathcal{A}^{i}, \mathcal{B}\right)$ for the mappings $f^{i}=\left.f\right|_{X^{i}}: X^{i} \rightarrow Y, i=1,2$. Clearly, if $(\mathcal{A}, \mathcal{B})$ is decomposable, the total space $X$ cannot be connected. We say that an overlay structure $[\mathcal{A}, \mathcal{B}]$ for a mapping $f: X \rightarrow Y$ is decomposable provided it contains a decomposable overlay pair. We say that an overlay mapping is decomposable provided it admits a decomposable overlay structure. Fox has exhibited examples of indecomposable overlay mappings $f: X \rightarrow Y$ between metric spaces, where the total space $X$ is not connected (see [2]). In those examples the number of sheets was infinite.

Now, we can state the main result of this section.

Theorem 2. Let $(\mathcal{A}, \mathcal{B})$ be an overlay pair for an s-sheeted overlay mapping $f: X \rightarrow$ $Y$ over a connected paracompact space $Y$ and let the number of sheets $s$ be finite. $(\mathcal{A}, \mathcal{B})$ is indecomposable if and only if $X$ is connected.

The proof of Theorem 2 is based on the following three lemmas.

Lemma 1. Let $f: X \rightarrow Y$ be an s-sheeted overlay mapping over a connected, paracompact space $Y$ and let $(\mathcal{A}, \mathcal{B})$ be an overlay pair for $f$. If the number of sheets 
$s$ is finite, then for every open covering $\mathcal{U}$ of $X$ there exists an open covering $\mathcal{V}$ of $Y$ such that $\mathcal{V} \leq \mathcal{B}$ and $f^{-1}(\mathcal{V}) \cap \mathcal{A} \leq \mathcal{U}$.

Proof. For each $y \in Y$ choose an arbitrary member $B_{y} \in \mathcal{B}$ such that $y \in B_{y}$. Let $\mathcal{W}$ be an open covering of $X$ such that $\mathrm{St} \mathcal{W} \leq \mathcal{U}$. For each point $x^{\sigma}=f^{-1}(y) \cap A_{B_{y}}^{\sigma}$, $\sigma \in S$, and $W_{y}^{\sigma}=\operatorname{St}\left(x^{\sigma}, \mathcal{W}\right) \cap A_{B_{y}}^{\sigma}$, put $V_{y}=\bigcap_{\sigma \in S} f\left(W_{y}^{\sigma}\right)$. Since $s$ is finite and $f$ is an open mapping, $V_{y}$ is a well-defined open set and $y \in V_{y} \subseteq B_{y}$. Let $\mathcal{V}=\left\{V_{y}: y \in Y\right\}$. Then $\mathcal{V}$ is an open covering of $Y$, which refines $\mathcal{B}$. We claim that $f^{-1}(\mathcal{V}) \cap \mathcal{A} \leq \mathcal{U}$. Let $f^{-1}\left(V_{y}\right) \cap A_{B}^{\sigma}$ be an arbitrary nonempty member of the covering $f^{-1}(\mathcal{V}) \bar{\cap}$. Then $\emptyset \neq f\left(f^{-1}\left(V_{y}\right) \cap A_{B}^{\sigma}\right) \subseteq V_{y} \cap B \subseteq B_{y} \cap B$. Since $(\mathcal{A}, \mathcal{B})$ is an overlay pair, for a given $\sigma \in S$, there exists a unique $\tau \in S$ such that $A_{B_{y}}^{\tau} \cap A_{B}^{\sigma} \neq \emptyset$. Now, we conclude that $f^{-1}\left(V_{y}\right) \cap A_{B}^{\sigma}=f^{-1}\left(\bigcap_{\lambda \in S} f\left(W_{y}^{\lambda}\right)\right) \cap A_{B}^{\sigma} \subseteq\left(\bigcup_{\lambda \in S} \operatorname{St}\left(x^{\lambda}, \mathcal{W}\right) \cap A_{B_{y}}^{\lambda}\right) \cap A_{B}^{\sigma} \subseteq$ $\bigcup_{\lambda \in S} \operatorname{St}\left(x^{\lambda}, \mathcal{W}\right) \cap A_{B_{y}}^{\lambda} \cap A_{B}^{\sigma} \subseteq \operatorname{St}\left(x^{\tau}, \mathcal{W}\right) \cap A_{B_{y}}^{\tau} \cap A_{B}^{\sigma} \subseteq \operatorname{St}\left(x^{\tau}, \mathcal{W}\right)$.

Since St $\mathcal{W} \leq \mathcal{U}$, there exists a $U \in \mathcal{U}$ such that $f^{-1}\left(V_{y}\right) \cap A_{B}^{\sigma} \subseteq U$, which proves our claim.

Note that from Lemma 1 easily follows that, for a finite-sheeted covering mapping $f: X \rightarrow Y$ over a connected compact space $Y$, the total space $X$ is also a compact space.

Lemma 2. Let

$$
\begin{array}{rll}
X_{0} & \stackrel{p}{\longleftarrow} & X \\
f_{0} \downarrow & & \downarrow f \\
Y_{0} & \longleftarrow & \\
& & Y
\end{array}
$$

be a pull-back diagram.

(i) Then $f_{0}^{-1}\left(f_{0}(p(X))\right)=p(X)$.

(ii) If $f_{0}: X_{0} \rightarrow Y_{0}$ is a covering mapping with a covering pair $(\mathcal{A}, \mathcal{B})$, then for each open covering $\mathcal{V}$ of $Y$ which refines $\mathcal{B}$, one has $\operatorname{St}\left(p(X), f_{0}^{-1}(\mathcal{V}) \cap \mathcal{A}\right)=$ $\operatorname{St}\left(p(X), f_{0}^{-1}(\mathcal{V})\right)$.

Proof. ( $i)$ We need to prove that $f_{0}^{-1}\left(f_{0}(p(X))\right) \subseteq p(X)$, since the other inclusion is always valid. Let $x_{0} \in f_{0}^{-1}\left(f_{0}(p(X))\right)$. Then $y_{0}=f_{0}\left(x_{0}\right) \in f_{0}(p(X))$. Therefore, there exists an $x \in X$ such that $y_{0}=f_{0}\left(x_{0}\right)=f_{0} p(x)=q f(x)$. By the assumption (1) is a pull-back diagram, and thus, $\left.p\right|_{f^{-1}(f(x))}: f^{-1}(f(x)) \rightarrow f_{0}^{-1}(q f(x))$ is a bijection (Lemma 6 of [5]). Since $x_{0} \in f_{0}^{-1}(q f(x))$, there exists a unique $x^{\prime} \in f^{-1}(f(x))$ such that $p\left(x^{\prime}\right)=x_{0}$. Consequently, $x_{0} \in p(X)$.

(ii) Since $f_{0}^{-1}(\mathcal{V}) \cap \mathcal{A} \leq f_{0}^{-1}(\mathcal{V})$, it is sufficient to prove $\operatorname{St}\left(p(X), f_{0}^{-1}(\mathcal{V})\right) \subseteq$ $\operatorname{St}\left(p(X), f_{0}^{-1}(\mathcal{V}) \cap \mathcal{A}\right)$. Let $x_{0} \in \operatorname{St}\left(p(X), f_{0}^{-1}(\mathcal{V})\right)$. This means that there exists a $V \bar{\epsilon}$ $\mathcal{V}$ such that $x_{0} \in f_{0}^{-1}(V)$ and $f_{0}^{-1}(V) \cap p(X) \neq \emptyset$. Let $x_{0}^{\prime} \in f_{0}^{-1}(V) \cap p(X)$ and $x^{\prime} \in X$ 
be such points that $x_{0}^{\prime}=p\left(x^{\prime}\right) \in f_{0}^{-1}(V)$. Now, we get $f_{0}\left(x_{0}\right), f_{0} p\left(x^{\prime}\right)=q f\left(x^{\prime}\right) \in V$. Let $B \in \mathcal{B}$ be a member of the covering $\mathcal{B}$ such that $f_{0}\left(x_{0}\right), f_{0} p\left(x^{\prime}\right) \in V \subseteq B$. There are unique $\sigma \in S$ and unique $\tau \in S$ such that $x_{0} \in A_{B}^{\sigma}$ and $p\left(x^{\prime}\right) \in A_{B}^{\tau}$. If $\sigma=\tau$, then $x_{0}, p\left(x^{\prime}\right) \in f_{0}^{-1}(V) \cap A_{B}^{\sigma}$. Therefore, $x_{0} \in \operatorname{St}\left(p(X), f_{0}^{-1}(\mathcal{V}) \cap \mathcal{A}\right)$. Assume that $\sigma \neq \tau$. Let $x_{0}^{\prime \prime} \in A_{B}^{\sigma}$ such that $f_{0}\left(x_{0}^{\prime \prime}\right)=f_{0}\left(p\left(x^{\prime}\right)\right)=q f\left(x^{\prime}\right)$. Since $\left.p\right|_{f^{-1}\left(f\left(x^{\prime}\right)\right)}: f^{-1}\left(f\left(x^{\prime}\right)\right) \rightarrow f_{0}^{-1}\left(q f\left(x^{\prime}\right)\right)$ is a bijection, there exists a unique point $x^{\prime \prime} \in X$ such that $x_{0}^{\prime \prime}=p\left(x^{\prime \prime}\right)$ and $x^{\prime \prime} \in f^{-1}\left(f\left(x^{\prime}\right)\right)$. So, $x_{0}^{\prime \prime}, x_{0} \in A_{B}^{\sigma}$ and $f_{0}\left(x_{0}^{\prime \prime}\right)=$ $f_{0}\left(p\left(x^{\prime}\right)\right) \in V$. Therefore, $x_{0}^{\prime \prime}, x_{0} \in A_{B}^{\sigma} \cap f_{0}^{-1}(V)$. Since $x_{0}^{\prime \prime}=p\left(x^{\prime \prime}\right)$, it follows that $x_{0} \in \operatorname{St}\left(p(X), f_{0}^{-1}(\mathcal{V}) \cap \mathcal{A}\right)$.

In the next lemma we shall need the notion of an ANR-resolution of a space and the notion of an ANR-overlay-enriched pull-back expansion of an s-sheeted overlay mapping $f: X \rightarrow Y$ over a connected space $Y$. Let us first recall these notions following [5].

Let $\boldsymbol{Y}=\left(Y_{\lambda}, q_{\lambda \lambda^{\prime}}, \Lambda\right)$ be an inverse system of topological spaces and let $\boldsymbol{q}=\left(q_{\lambda}\right)$ : $Y \rightarrow \boldsymbol{Y}$ be a mapping of a space $Y$. According to [4] (also see [6]), $\boldsymbol{q}$ is a resolution of $Y$ if it has the following two properties:

(R1) For every ANR $P$, every open covering $\mathcal{V}$ of $P$ and every mapping $f: Y \rightarrow P$, there exist a $\lambda \in \Lambda$ and a mapping $h: Y_{\lambda} \rightarrow P$ such that the mappings $h q_{\lambda}$ and $f$ are $\mathcal{V}$-near, i.e., every $x \in X$ admits a $V \in \mathcal{V}$ such that $h q_{\lambda}(x), f(x) \in V$;

(R2) For every ANR $P$ and every open covering $\mathcal{V}$ of $P$, there exists an open covering $\mathcal{V}^{\prime}$ of $P$, such that whenever, for a $\lambda \in \Lambda$ and for two mappings $h, h^{\prime}: Y_{\lambda} \rightarrow P$, the mappings $h q_{\lambda}, h^{\prime} q_{\lambda}$ are $\mathcal{V}^{\prime}$-near, then there exists a $\lambda^{\prime} \geq \lambda$, such that the mappings $h q_{\lambda \lambda^{\prime}}, h^{\prime} q_{\lambda \lambda^{\prime}}$ are $\mathcal{V}$-near.

If in a resolution $\boldsymbol{q}: Y \rightarrow \boldsymbol{Y}$ all the spaces $Y_{\lambda}$ are ANR's (for metric spaces), we speak of an ANR-resolution of $Y$. It is a well known fact that every topological space admits an ANR-resolution (see [4]). Moreover, if $Y$ is connected, one can achieve that all $Y_{\lambda}$ are connected ANR's. It is also well known that an inverse limit $\boldsymbol{q}: Y \rightarrow \boldsymbol{Y}$ is a resolution whenever $Y$ and all $Y_{\lambda}$ are compact Hausdorff spaces. Conversely, if all $Y_{\lambda}$ are Tychonoff spaces and $Y$ is topologically complete (e.g., paracompact), then every resolution $\boldsymbol{q}: Y \rightarrow \boldsymbol{Y}$ is an inverse limit (see e.g., [6]). It is very useful to know that properties (R1) and (R2) are equivalent to the following properties (B1) and (B2) (see e.g., [6]):

(B1) For every normal covering $\mathcal{U}$ of $Y$, there exist a $\lambda \in \Lambda$ and an open covering $\mathcal{V}$ of $Y_{\lambda}$ such that $q_{\lambda}^{-1} \mathcal{V} \leq \mathcal{U}$;

(B2) For every $\lambda \in \Lambda$ and every open covering $\mathcal{V}$ of $Y_{\lambda}$, there exists a $\lambda^{\prime} \geq \lambda$, such that $q_{\lambda \lambda^{\prime}}\left(Y_{\lambda^{\prime}}\right) \subseteq \operatorname{St}\left(q_{\lambda}(Y), \mathcal{V}\right)$.

We shall now recall the notion of an ANR-pull-back expansion of an $s$-sheeted overlay mapping $f: X \rightarrow Y$, denoted by $\boldsymbol{E}$ (see [5]). It consists of $f$, of an ANRresolution $\boldsymbol{q}=\left(q_{\lambda}\right): Y \rightarrow \boldsymbol{Y}$, of a mapping $\boldsymbol{p}=\left(p_{\lambda}\right): X \rightarrow \boldsymbol{X}$ and of a mapping 
$\boldsymbol{f}=\left(f_{\lambda}\right): \boldsymbol{X} \rightarrow \boldsymbol{Y}$, such that $\boldsymbol{f} \boldsymbol{p}=\boldsymbol{q} f$ and the following diagrams $D_{\lambda}, \lambda \in \Lambda$, are pull-back diagrams

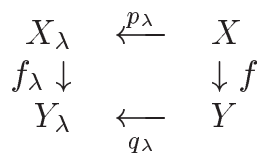

Moreover, for $\lambda \leq \lambda^{\prime}$, similar diagrams $D_{\lambda \lambda^{\prime}}$ consisting of the mappings $f_{\lambda}, f_{\lambda^{\prime}}, p_{\lambda \lambda^{\prime}}$ and $q_{\lambda \lambda^{\prime}}$ are also pull-back diagrams. Furthermore, we require that the mappings $f_{\lambda}: X_{\lambda} \rightarrow Y_{\lambda}, \lambda \in \Lambda$, be $s$-sheeted covering mappings. If $Y$ is connected we require that all the ANRs $Y_{\lambda}$ also be connected. If all mappings appearing in the ANR-pullback expansion $\boldsymbol{E}$ are pointed mappings, we shall say that the expansion is pointed and we shall use the notation $\boldsymbol{E}_{*}$.

We also recall (see [5]) the notion of an ANR- overlay-enriched pull-back expansion of an $s$-sheeted overlay mapping $f: X \rightarrow Y$ over a connected space $Y$, abbreviated to ANR- enriched pull-back expansion and denoted by $E \boldsymbol{E}$. It consists of an underlying ANR-pull-back expansion $\boldsymbol{E}$ for $f$ together with an $s$-sheeted overlay pair $(\mathcal{A}, \mathcal{B})$ for $f, s$-sheeted overlay pairs $\left(\mathcal{A}_{\lambda}, \mathcal{B}_{\lambda}\right)$ for $f_{\lambda}$ and some functions $\beta_{\lambda}: \mathcal{B}_{\lambda} \rightarrow \mathcal{B}$, which complete the pull-back diagrams $D_{\lambda}$ to enriched pull-back diagrams $E D_{\lambda}$. The latter condition imposes some additional requirements (see section 4 of [5]). For our consideration the only important additional requirement is that $q_{\lambda}^{-1}\left(\mathcal{B}_{\lambda}\right)$ refines $\mathcal{B}$ and $p_{\lambda}^{-1}\left(\mathcal{A}_{\lambda}\right)$ refines $\mathcal{A}$, for each $\lambda \in \Lambda$. If all mappings appearing in the ANR-enriched pull-back expansion are pointed mappings, we shall say that the expansion is pointed and we shall use the notation $E \boldsymbol{E}_{*}$.

Lemma 3. Let $(\mathcal{A}, \mathcal{B})$ be an overlay pair for an s-sheeted overlay mapping $f: X \rightarrow Y$ over a connected paracompact space $Y$ and let $E \boldsymbol{E}$ be an ANR-overlay-enriched pullback expansion asssociated with $f$ and $(\mathcal{A}, \mathcal{B})$. If the number of sheets $s$ is finite, then $\boldsymbol{p}=\left(p_{\lambda}\right): X \rightarrow \boldsymbol{X}=\left(X_{\lambda}, p_{\lambda \lambda^{\prime}}, \Lambda\right)$ is a resolution of $X$.

Proof. Since $\boldsymbol{p}=\left(p_{\lambda}\right): X \rightarrow \boldsymbol{X}=\left(X_{\lambda}, p_{\lambda \lambda^{\prime}}, \Lambda\right)$ is a mapping of $X$, we need to prove (B1) and (B2).

(B1) Let $\mathcal{U}$ be an arbitrary open covering of $X$. According to Lemma 1, there exists an open covering $\mathcal{V}$ of $Y$ such that $\mathcal{V} \leq \mathcal{B}$ and $f^{-1}(\mathcal{V}) \cap \mathcal{A} \leq \mathcal{U}$. Since $\boldsymbol{q}=\left(q_{\lambda}\right): Y \rightarrow \boldsymbol{Y}=\left(Y_{\lambda}, q_{\lambda \lambda^{\prime}}, \Lambda\right)$ is a resolution of $Y$, there exist a $\lambda \in \Lambda$ and an open covering $\mathcal{V}_{\lambda}$ of $Y_{\lambda}$ such that $q_{\lambda}^{-1}\left(\mathcal{V}_{\lambda}\right) \leq \mathcal{V}$. Let $\left(\mathcal{A}_{\lambda}, \mathcal{B}_{\lambda}\right)$ be the overlay pair for $f_{\lambda}: X_{\lambda} \rightarrow Y_{\lambda}$ given by $E \boldsymbol{E}$. Without loss of generality we may assume that $\mathcal{V}_{\lambda} \leq \mathcal{B}_{\lambda}$. Let us now consider the open covering $f_{\lambda}^{-1}\left(\mathcal{V}_{\lambda}\right) \cap \mathcal{A}_{\lambda}$ of $X_{\lambda}$. We claim that $p_{\lambda}^{-1}\left(f_{\lambda}^{-1}\left(\mathcal{V}_{\lambda}\right) \cap \mathcal{A}_{\lambda}\right) \leq \mathcal{U}$. Indeed,

$p_{\lambda}^{-1}\left(f_{\lambda}^{-1}\left(\mathcal{V}_{\lambda}\right) \cap \mathcal{A}_{\lambda}\right)=p_{\lambda}^{-1}\left(f_{\lambda}^{-1}\left(\mathcal{V}_{\lambda}\right)\right) \cap p_{\lambda}^{-1}\left(\mathcal{A}_{\lambda}\right) \leq\left(f_{\lambda} p_{\lambda}\right)^{-1}\left(\mathcal{V}_{\lambda}\right) \cap \mathcal{A}=$ $\left(q_{\lambda} f\right)^{-1}\left(\mathcal{V}_{\lambda}\right) \cap \mathcal{A}=f^{-1}\left(q_{\lambda}^{-1}\left(\mathcal{V}_{\lambda}\right)\right) \cap \mathcal{A} \leq f^{-1}(\mathcal{V}) \cap \mathcal{A} \leq \mathcal{U}$

This proves (B1).

(B2) Let $\mathcal{U}$ be an arbitrary open covering of $X_{\lambda}, \lambda \in \Lambda$. According to Lemma 1 , there exists an open covering $\mathcal{V}$ of $Y_{\lambda}$ such that $\mathcal{V} \leq \mathcal{B}_{\lambda}$ and $f_{\lambda}^{-1}(\mathcal{V}) \cap \mathcal{A}_{\lambda} \leq \mathcal{U}$. 
Since $\boldsymbol{q}=\left(q_{\lambda}\right): Y \rightarrow \boldsymbol{Y}=\left(Y_{\lambda}, q_{\lambda \lambda^{\prime}}, \Lambda\right)$ is a resolution of $Y$ there exists a $\lambda^{\prime} \geq \lambda$ such that $q_{\lambda \lambda^{\prime}}\left(Y_{\lambda^{\prime}}\right) \subseteq \operatorname{St}\left(q_{\lambda}(Y), \mathcal{V}\right)$. This implies that $q_{\lambda \lambda^{\prime}}\left(f_{\lambda^{\prime}}\left(X_{\lambda^{\prime}}\right)\right) \subseteq \operatorname{St}\left(q_{\lambda}(f(X)), \mathcal{V}\right)$, i.e., $f_{\lambda} p_{\lambda \lambda^{\prime}}\left(X_{\lambda^{\prime}}\right) \subseteq \operatorname{St}\left(f_{\lambda} p_{\lambda}(X), \mathcal{V}\right)$. So, using Lemma 2 (i) and (ii) we get

$$
\begin{aligned}
& p_{\lambda \lambda^{\prime}}\left(X_{\lambda^{\prime}}\right) \subseteq f_{\lambda}^{-1}\left(\operatorname{St}\left(f_{\lambda} p_{\lambda}(X), \mathcal{V}\right)\right) \subseteq \operatorname{St}\left(f_{\lambda}^{-1}\left(f_{\lambda} p_{\lambda}(X)\right), f_{\lambda}^{-1}(\mathcal{V})\right)= \\
& =\operatorname{St}\left(p_{\lambda}(X), f_{\lambda}^{-1}(\mathcal{V})\right)=\operatorname{St}\left(p_{\lambda}(X), f_{\lambda}^{-1}(\mathcal{V}) \cap \mathcal{A}_{\lambda}\right) \subseteq \operatorname{St}\left(p_{\lambda}(X), \mathcal{U}\right),
\end{aligned}
$$

which proves (B2).

Proof of Theorem 2. We only need to prove that indecomposability of $(\mathcal{A}, \mathcal{B})$ implies connectedness of $X$, because connectedness of $X$ always implies indecomposability of $(\mathcal{A}, \mathcal{B})$. Let $\boldsymbol{q}=\left(q_{\lambda}\right): Y \rightarrow \boldsymbol{Y}$ be an ANR-resolution of $Y$ consisting of connected ANRs $Y_{\lambda}$. Then there exists a $\lambda_{0} \in \Lambda$ such that $(\mathcal{A}, \mathcal{B})$ and the restriction of $\boldsymbol{q}=\left(q_{\lambda}\right): Y \rightarrow \boldsymbol{Y}$ to the subset $\Lambda_{0}=\left\{\lambda \in \Lambda: \lambda \geq \lambda_{0}\right\} \subseteq \Lambda$ can be completed to an ANR-enriched pull-back expansion $E \boldsymbol{E}$ (Lemma 23 of [5]). Since $(\mathcal{A}, \mathcal{B})$ is indecomposable, all the mappings $f_{\lambda}: X_{\lambda} \rightarrow Y_{\lambda}, \lambda \in \Lambda_{0}$, given by $E \boldsymbol{E}$ are indecomposable and all ANRs $X_{\lambda}, \lambda \in \Lambda_{0}$, are connected (Remark 7 and Remark 8 of [5]). Since the number of sheets $s$ is finite, Lemma 3 implies that $\boldsymbol{p}=\left(p_{\lambda}\right): X \rightarrow \boldsymbol{X}=\left(X_{\lambda}, p_{\lambda \lambda^{\prime}}, \Lambda_{0}\right)$ is a resolution of $X$. Since all $X_{\lambda}, \lambda \in \Lambda_{0}$, are connected, (R1) implies that $X$ is connected.

\section{Classification theorem for finite-sheeted covering mappings over con- nected paracompact spaces}

Let $[\mathcal{A}, \mathcal{B}]$ be a covering (overlay) structure for an $s$-sheeted covering (overlay) mapping $f: X \rightarrow Y$ and let $\left[\mathcal{A}^{\prime}, \mathcal{B}^{\prime}\right]$ be a covering (overlay) structure for another $s$ sheeted covering (overlay) mapping $f^{\prime}: X^{\prime} \rightarrow Y$ over the same connected space $Y$. We say that the two structures are equivalent if there exists a homeomorphism $\varphi: X \rightarrow X^{\prime}$ such that $f^{\prime} \varphi=f$ and the covering (overlay) structure $\left[(\varphi)^{-1}\left(\mathcal{A}^{\prime}\right), \mathcal{B}^{\prime}\right]$ coincides with $[\mathcal{A}, \mathcal{B}]$ (or equivalently, $[\varphi(\mathcal{A}), \mathcal{B}]$ coincides with $\left[\mathcal{A}^{\prime}, \mathcal{B}^{\prime}\right]$ ). A mapping of pointed spaces $f:(X, *) \rightarrow(Y, *)$ is a pointed covering mapping ( pointed overlay mapping) if $f: X \rightarrow Y$ is a covering (overlay) mapping. In the same way one defines pointed equivalence of structures of pointed mappings $f:(X, *) \rightarrow(Y, *)$. The only difference is the additional requirement that the homeomorphism $\varphi$ be a pointed mapping $\varphi:(X, *) \rightarrow\left(X^{\prime}, *\right)$.

Let $Y$ be a connected paracompact space and let $s$ be finite. We define a function $\left(\Psi_{*}\right) \Psi$ from (pointed ) $s$-sheeted covering mappings $(f:(X, *) \rightarrow(Y, *)) f: X \rightarrow$ $Y$ with a connected total space $X$ to (pointed) $s$-sheeted indecomposable overlay structures $\left([\mathcal{A}, \mathcal{B}]_{*}\right)[\mathcal{A}, \mathcal{B}]$ over $Y$. By Theorem 1 , each $s$-sheeted (pointed) covering mapping $f: X \rightarrow Y$ admits a unique (pointed) overlay structure $[\mathcal{A}, \mathcal{B}]$ and since the total space $X$ is connected, $[\mathcal{A}, \mathcal{B}]$ is indecomposable, by Theorem 2 . We put $\Psi(f)=[\mathcal{A}, \mathcal{B}]\left(\Psi_{*}(f)=[\mathcal{A}, \mathcal{B}]_{*}\right.$ for pointed $\left.f\right)$. Let (pointed) covering mappings $f$ : $X \rightarrow Y$ and $f^{\prime}: X^{\prime} \rightarrow Y$ be equivalent, i.e., there exists a (pointed) homeomorphism $\varphi: X \rightarrow X^{\prime}$ such that $f^{\prime} \varphi=f$. Then, by Theorem 1, the (pointed) overlay structures $\left(\Psi_{*}(f)=[\mathcal{A}, \mathcal{B}]_{*}\right) \Psi(f)=[\mathcal{A}, \mathcal{B}]$ and $\left(\Psi_{*}\left(f^{\prime}\right)=\left[\mathcal{A}^{\prime}, \mathcal{B}^{\prime}\right]_{*}\right) \Psi\left(f^{\prime}\right)=\left[\mathcal{A}^{\prime}, \mathcal{B}^{\prime}\right]$ 
are equivalent. This enables us to define a function $\left(\bar{\Psi}_{*}\right) \bar{\Psi}$ on the set of (pointed) equivalence classes by putting $\left(\bar{\Psi}_{*}\left([f]_{*}\right)=\left[\Psi_{*}(f)\right]\right) \bar{\Psi}[f]=[\Psi(f)]$. So, $\left(\bar{\Psi}_{*}: \mathfrak{C}_{*}^{s} \rightarrow\right.$ $\left.\mathfrak{O}_{*}^{s}\right) \bar{\Psi}: \mathfrak{C}^{s} \rightarrow \mathfrak{O}^{s}$ is the function from the set $\left(\mathfrak{C}_{*}^{s}\right) \mathfrak{C}^{s}$ of all (pointed) equivalence classes of $s$-sheeted covering mappings $f: X \rightarrow Y$ with a connected total space $X$ to the set $\left(\mathfrak{O}_{*}^{s}\right) \mathfrak{O}^{s}$ of all (pointed) equivalence classes of $s$-sheeted indecomposable overlay structures over $Y$. It is obvious that $\left(\bar{\Psi}_{*}\right) \bar{\Psi}$ is an injection. Namely, if (pointed) indecomposable $s$-sheeted overlay structures $[\mathcal{A}, \mathcal{B}]$ for $f: X \rightarrow Y$ and $\left[\mathcal{A}^{\prime}, \mathcal{B}^{\prime}\right]$ for $f^{\prime}$ : $X^{\prime} \rightarrow Y^{\prime}$ are equivalent, then by definition, there exists a (pointed) homeomophism $\varphi: X \rightarrow X^{\prime}$ such that $f^{\prime} \varphi=f$. However, this means that $f$ and $f^{\prime}$ are equivalent. Furthermore, $\left(\bar{\Psi}_{*}\right) \bar{\Psi}$ is a surjection, since by Theorem 2, indecomposability of an overlay structure $[\mathcal{A}, \mathcal{B}]$ for $f: X \rightarrow Y$ implies connectedness of $X$. So, we have proved the following theorem.

Theorem 3. Let $Y$ be a connected, paracompact space and let $s \in \mathbb{N}$. Then the function $\left(\bar{\Psi}_{*}: \mathfrak{C}_{*}^{s} \rightarrow \mathfrak{O}_{*}^{s}\right) \bar{\Psi}: \mathfrak{C}^{s} \rightarrow \mathfrak{O}^{s}$, which to each (pointed) equivalence class of a (pointed) s-sheeted covering mapping $(f:(X, *) \rightarrow(Y, *)) f: X \rightarrow Y$ with $X$ connected assigns a (pointed) equivalence class of the unique indecomposable overlay structure $\left([\mathcal{A}, \mathcal{B}]_{*}\right)[\mathcal{A}, \mathcal{B}]$ for $f$, is a bijection.

As we already mentioned in the Introduction, Mardešić and Matijević recently classified indecomposable overlay structures over arbitrary connected topological spaces. Their classification theorem establishes a bijection $\widetilde{\Phi}_{*}: \mathfrak{O}_{*}^{s} \rightarrow \mathfrak{P}_{*}^{s}$ between the set $\mathfrak{O}_{*}^{s}$ of all pointed equivalence classes of $s$-sheeted indecomposable overlay structures over connected topological space $(Y, *)$ and the set $\mathfrak{P}_{*}^{s}$ of all subprogroups of index $s$ of the fundamental progroup $\underline{\pi}_{1}(Y, *)$ (Theorem 1 of [5]). In the unpointed case it establishes a bijection $\widetilde{\Phi}: \mathfrak{O}^{s} \rightarrow \mathfrak{P}^{s}$ between the set $\mathfrak{O}^{s}$ of all equivalence classes of $s$-sheeted indecomposable overlay structures over connected topological space $Y$ and the set $\mathfrak{P}^{s}$ of all conjugacy classes of subprogroups of index $s$ of the fundamental progroup $\underline{\pi}_{1}(Y, *)$, where $*$ is an arbitrary point of $Y$ (Theorem 4 of [5]). Before briefly describing the functions $\widetilde{\Phi}_{*}$ and $\widetilde{\Phi}$, we recall the definitions of progroups, subprogroups of index $s$, conjugacy classes of subprogroups of index $s$ and the fundamental progroup.

In general, a progroup is an inverse system of groups, $\mathbf{G}=\left(G_{\lambda}, q_{\lambda \lambda^{\prime}}, \Lambda\right)$. We define a subprogroup of $\mathbf{G}$ as a progroup $\mathbf{H}=\left(H_{\lambda}, q_{\lambda \lambda^{\prime}}, \Lambda_{0}\right)$, where $\Lambda_{0}$ is a subset of $\Lambda$ such that $\lambda \in \Lambda_{0}$ and $\lambda \leq \lambda^{\prime}$ imply $\lambda^{\prime} \in \Lambda_{0}$. Moreover, $H_{\lambda}$ is a subgroup of $G_{\lambda}, \lambda \in \Lambda_{0}$, and $q_{\lambda \lambda^{\prime}}: H_{\lambda^{\prime}} \rightarrow H_{\lambda}$ now denotes the restriction of $q_{\lambda \lambda^{\prime}}: G_{\lambda^{\prime}} \rightarrow G_{\lambda}, \lambda \leq \lambda^{\prime}$ from $\Lambda_{0}$. Often $\Lambda_{0}$ will have an initial element $\lambda_{0}$, i.e., $\Lambda_{0}=\left\{\lambda \in \Lambda: \lambda \geq \lambda_{0}\right\}$. We consider two subprogroups $\mathbf{H}, \mathbf{H}^{\prime} \subseteq \mathbf{G}$ as equal, provided there exists an element $\lambda^{*} \in \Lambda_{0} \cap \Lambda^{\prime}{ }_{0}$ such that $H_{\lambda}=H_{\lambda}^{\prime}$, for $\lambda \geq \lambda^{*}$. Note that the quotient sets $G_{\lambda} / H_{\lambda}$ of the right cosets and the induced functions $r_{\lambda \lambda^{\prime}}: G_{\lambda^{\prime}} / H_{\lambda^{\prime}} \rightarrow G_{\lambda} / H_{\lambda}$ form an inverse system of pointed sets $\mathbf{G} / \mathbf{H}=\left(G_{\lambda} / H_{\lambda}, r_{\lambda \lambda^{\prime}}, \Lambda_{0}\right)$. We say that the subprogroup $\mathbf{H} \subseteq \mathbf{G}$ has index $s$, and we write $\{\mathbf{G}: \mathbf{H}\}=s$, provided there exists an index $\lambda^{1}$ such that for every $\lambda \geq \lambda^{1}$, the index $\left\{G_{\lambda}: H_{\lambda}\right\}=s$ and $r_{\lambda \lambda^{\prime}}$ is a bijection, for $\lambda^{\prime} \geq \lambda \geq \lambda^{1}$.

Subprogroups $\mathbf{H}=\left(H_{\lambda}, q_{\lambda \lambda^{\prime}}, \Lambda_{0}\right)$ and $\mathbf{H}^{\prime}=\left(H_{\lambda}^{\prime}, q_{\lambda \lambda^{\prime}}, \Lambda^{\prime}{ }_{0}\right)$ of a progroup $\mathbf{G}=$ 
$\left(G_{\lambda}, q_{\lambda \lambda^{\prime}}, \Lambda\right)$ are said to be conjugate subprogroups of $\mathbf{G}$ provided there is a $\lambda^{*} \in \Lambda_{0} \cap$ $\Lambda_{0}^{\prime}$ and there is a system of elements $g_{\lambda} \in G_{\lambda}, \lambda \geq \lambda^{*}$, such that $H_{\lambda}^{\prime}=g_{\lambda}^{-1} H_{\lambda} g_{\lambda}, \lambda \geq$ $\lambda^{*}$, and $q_{\lambda \lambda^{\prime}}\left(g_{\lambda^{\prime}}\right) \in H_{\lambda} g_{\lambda}, \lambda^{\prime} \geq \lambda \geq \lambda^{*}$.

To define the fundamental progroup $\underline{\pi}_{1}(Y, *)$ of a pointed space $(Y, *)$, one takes an ANR-resolution $\boldsymbol{q}:(Y, *) \rightarrow(\boldsymbol{Y}, *)=\left(\left(Y_{\lambda}, *\right), q_{\lambda \lambda^{\prime}}, \Lambda\right)$ of $(Y, *)$. Application of $\pi_{1}$ yields the progroup $\pi_{1}(\boldsymbol{Y}, *)=\left(\pi_{1}\left(Y_{\lambda}, *\right),\left(q_{\lambda \lambda^{\prime}}\right)_{\#}, \Lambda\right)$, which is by definition, the fundamental progroup $\underline{\pi}_{1}(Y, *)$. It is independent (up to natural isomorphism) of the choice of the ANR-resolution (see II.3.3 of [6]).

First we define the function $\Phi_{*}$ from $s$-sheeted indecomposable overlay pairs $(\mathcal{A}, \mathcal{B})$, for pointed overlay mappings $f:(X, *) \rightarrow(Y, *)$, to subprogroups of index $s$ of $\underline{\pi}_{1}(Y, *)$ in the following way. Associate with a connected space $Y$ a fixed resolution $\mathbf{q}:(Y, *) \rightarrow(\mathbf{Y}, *)$, which consists of connected $\operatorname{ANR}$ 's $\left(Y_{\lambda}, *\right)$. For a given indecomposable overlay pair $(\mathcal{A}, \mathcal{B})$ for a pointed $s$-sheeted overlay mapping $f:(X, *) \rightarrow(Y, *)$, there exists a subset $\Lambda_{0} \subseteq \Lambda$ such that the restriction $\boldsymbol{q}^{0}$ of $\boldsymbol{q}$ to $\Lambda_{0}$ can be completed to a pointed ANR-enriched pull-back expansion $E \boldsymbol{E}_{*}$. For each $\lambda \in \Lambda_{0}$, put $H_{\lambda}=f_{\lambda \#}\left(\pi_{1}\left(X_{\lambda}, *\right)\right)$. Then $\mathbf{H}_{*}=\left\{H_{\lambda}, q_{\lambda \lambda^{\prime} \#}, \Lambda_{0}\right\}$ is a subprogroup of index $s$ of the fundamental progroup $\mathbf{G}=\underline{\pi}_{1}(Y, *)$ (see Sections 5 and 6 of [5] ). Now, put $\Phi_{*}(\mathcal{A}, \mathcal{B})=\mathbf{H}_{*}$.

Since $\Phi_{*}$ depends only on the overlay structure $[\mathcal{A}, \mathcal{B}]$ of $(\mathcal{A}, \mathcal{B})$, it enables us to define a function $\bar{\Phi}_{*}$ by putting $\bar{\Phi}_{*}[\mathcal{A}, \mathcal{B}]=\Phi_{*}(\mathcal{A}, \mathcal{B})$. Furthemore, $\bar{\Phi}_{*}$ depends only on the equivalence class of $[\mathcal{A}, \mathcal{B}]$ and therefore, we can define $\widetilde{\Phi}_{*}$ on the class of $[\mathcal{A}, \mathcal{B}]$ as $\bar{\Phi}_{*}[\mathcal{A}, \mathcal{B}] . \widetilde{\Phi}_{*}: \mathfrak{O}_{*}^{s} \rightarrow \mathfrak{P}_{*}^{s}$ is a well-defined bijection (see Lemmas 25, 26 and 27 of $[5])$.

In the unpointed case we define the function $\widetilde{\Phi}: \mathfrak{O}^{s} \rightarrow \mathfrak{P}^{s}$ as follows. Let $*$ be an arbitrarily chosen point of $Y$. Let $(\mathcal{A}, \mathcal{B})$ be an indecomposable overlay pair for an $s$-sheeted overlay mapping $f: X \rightarrow Y$. Choose an arbitrary point $* \in f^{-1}(*)$. Now, we can consider $(\mathcal{A}, \mathcal{B})$ as a pointed indecomposable overlay pair for an $s$-sheeted pointed overlay mapping $f:(X, *) \rightarrow(Y, *)$. Let $\widetilde{\Phi}$ assign to an equivalence class of $[\mathcal{A}, \mathcal{B}]$ the conjugacy class of $\mathbf{H}_{*}$, which is the image of the pointed equivalence class of $[\mathcal{A}, \mathcal{B}]$ by $\widetilde{\Phi}_{*} . \widetilde{\Phi}$ is a well-defined bijection (see Section 10 of [5]).

Let us now consider the compositions $F_{*}=\widetilde{\Phi}_{*} \bar{\Psi}_{*}: \mathfrak{C}_{*}^{s} \rightarrow \mathfrak{P}_{*}^{s}$ and $F=\widetilde{\Phi} \bar{\Psi}$ : $\mathfrak{C}^{s} \rightarrow \mathfrak{P}^{s}$. Note that $F_{*}\left([f]_{*}\right)=\widetilde{\Phi}_{*} \bar{\Psi}_{*}\left([f]_{*}\right)=\widetilde{\Phi}_{*}\left[\Psi_{*}(f)\right]=\bar{\Phi}_{*}\left(\Psi_{*}(f)\right)$ and $F([f])=$ $\widetilde{\Phi} \bar{\Psi}([f])=\left[\widetilde{\Phi}_{*} \bar{\Psi}_{*}\left([f]_{*}\right)\right]=\left[F_{*}\left([f]_{*}\right)\right]$. Since $F_{*}$ and $F$ are bijections, we get the following two classification theorems.

Theorem 4. Let $(Y, *)$ be a connected, paracompact space and let $s \in \mathbb{N}$. Then $F_{*}$ establishes a bijection between the set of all pointed equivalence classes of s-sheeted pointed covering mappings $f:(X, *) \rightarrow(Y, *)$ with a connected total space $X$ and the set of all subprogroups of index $s$ of the fundamental progroup $\underline{\pi}_{1}(Y, *)$.

Theorem 5. Let $Y$ be a connected, paracompact space and let $s \in \mathbb{N}$. Then $F$ establishes a bijection between the set of all equivalence classes of s-sheeted pointed 
covering mappings $f: X \rightarrow Y$ with a connected total space $X$ and the set of all conjugacy classes of subprogroups of index $s$ of the fundamental progroup $\underline{\pi}_{1}(Y, *)$, where $*$ is an arbitrarily chosen point of $Y$.

The next theorem gives an explicit procedure to detect whether a pointed mapping $f:(X, *) \rightarrow(Y, *)$ is an $s$-sheeted covering with a connected total space $(X, *)$ and in that case, it also gives the subprogroup $F_{*}\left([f]_{*}\right)$.

Theorem 6. Let $Y$ be a connected paracompact space, let $f:(X, *) \rightarrow(Y, *)$ be a pointed mapping and let $s \in \mathbb{N}$. Let $\boldsymbol{q}=\left(q_{\lambda}, \lambda \in \Lambda\right): Y \rightarrow \boldsymbol{Y}$ be an arbitrary resolution of $Y$ consisting of connected ANRs. $f$ is a pointed s-sheeted covering mapping with connected total space if and only if there exists a pointed mapping $\boldsymbol{f}_{*}=\left(f_{\lambda}:\left(X_{\lambda}, *\right) \rightarrow\left(Y_{\lambda}, *\right), \lambda \in \Lambda_{0}\right): \boldsymbol{X} \rightarrow \boldsymbol{Y}^{\mathbf{0}}$ over some subset $\Lambda_{0}=\left\{\lambda \in \Lambda: \lambda \geq \lambda_{0}\right\}$, such that each mapping $f_{\lambda}:\left(X_{\lambda}, *\right) \rightarrow\left(Y_{\lambda}, *\right)$ is an $s$ sheeted covering mapping with connected total space, for every $\lambda \leq \lambda^{\prime}$, pointed mappings $f_{\lambda}, f_{\lambda^{\prime}}, p_{\lambda \lambda^{\prime}}$ and $q_{\lambda \lambda^{\prime}}$ form pointed pull-back diagrams $D_{\lambda \lambda^{\prime}}$ and $f=\lim f_{*}$. In that case $F_{*}\left([f]_{*}\right)=\mathbf{H}_{*}=\left\{H_{\lambda}, q_{\lambda \lambda^{\prime} \#}, \Lambda_{1}\right\}$, where $H_{\lambda}=f_{\lambda \#}\left(\pi_{1}\left(X_{\lambda}, *\right)\right)$ and $\Lambda_{1}=\left\{\lambda \in \Lambda_{0}: \lambda \geq \lambda_{1}\right\} \subseteq \Lambda_{0}$ for some $\lambda_{1} \in \Lambda_{0}$.

Proof. Let $f:(X, *) \rightarrow(Y, *)$ be a pointed $s$-sheeted covering mapping with connected total space $X$. Since $Y$ is paracompact, then also $X$ is paracompact. According to Theorems 1 and $2, f$ admits an $s$-sheeted indecomposable overlay pair $(\mathcal{A}, \mathcal{B})$ and $\Psi_{*}(f)=[\mathcal{A}, \mathcal{B}]_{*}$. Then there exists a $\lambda_{0} \in \Lambda$ such that $(\mathcal{A}, \mathcal{B})$ and the restriction $\boldsymbol{q}^{\mathbf{0}}: Y \rightarrow \boldsymbol{Y}^{\mathbf{0}}$ of $\boldsymbol{q}=\left(q_{\lambda}\right): Y \rightarrow \boldsymbol{Y}$ to the subset $\Lambda_{0}=\left\{\lambda \in \Lambda: \lambda \geq \lambda_{0}\right\} \subseteq \Lambda$ can be completed to a pointed ANR-enriched pull-back expansion $E \boldsymbol{E}_{*}$ (Lemma 23 of [5]). Since $(\mathcal{A}, \mathcal{B})$ is indecomposable, all pointed $s$-sheeted covering mappings $f_{\lambda}: X_{\lambda} \rightarrow Y_{\lambda}, \lambda \in \Lambda_{0}$, given by $E \boldsymbol{E}_{*}$ are indecomposable $s$-sheeted covering mappings, and therefore, all ANRs $X_{\lambda}, \lambda \in \Lambda_{0}$, are connected (Remark 6 and Remark 7 of [5]). Also, note that all pointed diagrams $D_{\lambda \lambda^{\prime}}, \lambda^{\prime} \geq \lambda \geq \lambda_{0}$ are pull-back diagrams. Since the number of sheets $s$ is finite, Lemma 3 implies that $\boldsymbol{p}=\left(p_{\lambda}\right): X \rightarrow \boldsymbol{X}=\left(X_{\lambda}, p_{\lambda \lambda^{\prime}}, \Lambda_{0}\right)$ is a resolution of $X$. Moreover, $X$ is the limit of $\boldsymbol{X}$, because $X$ is paracompact. Also, $\boldsymbol{q}^{\mathbf{0}}: Y \rightarrow \boldsymbol{Y}^{\mathbf{0}}$ is the limit, since $Y$ is paracompact and $\Lambda_{0}$ cofinal in $\Lambda$. Consequently, $f$ is the limit of $f_{*}$ appearing in $E \boldsymbol{E}_{*}$ and $\boldsymbol{f}_{*}$ has all the desired properties. Furthermore, $\bar{\Phi}_{*}\left([\mathcal{A}, \mathcal{B}]_{*}\right)=\mathbf{H}_{*}=\left\{H_{\lambda}, q_{\lambda \lambda^{\prime} \#}, \Lambda_{0}\right\}$, where $H_{\lambda}=f_{\lambda \#}\left(\pi_{1}\left(X_{\lambda}, *\right)\right)$. Since, $F_{*}\left([f]_{*}\right)=\bar{\Phi}_{*}\left(\Psi_{*}(f)\right)=\bar{\Phi}_{*}\left([\mathcal{A}, \mathcal{B}]_{*}\right)=\mathbf{H}_{*}$, necessity is proved.

Let us prove sufficiency. Assume that a pointed mapping $f:(X, *) \rightarrow(Y, *)$ over the connected paracompact space $Y$ is the limit of a pointed mapping $\boldsymbol{f}_{*}=\left(f_{\lambda}\right.$ : $\left.\left(X_{\lambda}, *\right) \rightarrow\left(Y_{\lambda}, *\right), \lambda \in \Lambda_{0}\right): \boldsymbol{X} \rightarrow \boldsymbol{Y}^{0}$ over some subset $\Lambda_{0}=\left\{\lambda \in \Lambda: \lambda \geq \lambda_{0}\right\}$ of $\Lambda$, such that, each mapping $f_{\lambda}:\left(X_{\lambda}, *\right) \rightarrow\left(Y_{\lambda}, *\right)$ is a pointed $s$-sheeted covering mapping with a connected total space and all diagrams $D_{\lambda \lambda^{\prime}}, \lambda^{\prime} \geq \lambda \geq \lambda_{0}$, are pullback diagrams. Since $X=\lim \boldsymbol{X}, Y=\lim \boldsymbol{Y}^{0}$ and $f=\lim \boldsymbol{f}_{*}$, all the diagrams $D_{\lambda}$ consisting of pointed mappings $f_{\lambda}, f, p_{\lambda}$ and $q_{\lambda}$ are pull-back diagrams (see Lemma 12 of [5]). Consequently, $f$ is an $s$-sheeted covering mapping (see Lemma 13 of [5]). Using 
some properties of the resolution $\boldsymbol{q}$, it is possible to find $\lambda_{1} \in \Lambda_{0}$ and an $s$-sheeted indecomposable overlay pair $\left(\mathcal{A}_{\lambda_{1}}, \mathcal{B}_{\lambda_{1}}\right)$ of $f_{\lambda_{1}}:\left(X_{\lambda_{1}}, *\right) \rightarrow\left(Y_{\lambda_{1}}, *\right)$ such that the $s$-sheeted indecomposable overlay pairs $\left(\mathcal{A}_{\lambda}, \mathcal{B}_{\lambda}\right)=\left(p_{\lambda_{1} \lambda}^{-1} \mathcal{A}_{\lambda_{1}}, q_{\lambda_{1} \lambda}^{-1} \mathcal{B}_{\lambda_{1}}\right), \lambda \geq \lambda_{1}$, and $(\mathcal{A}, \mathcal{B})=\left(p_{\lambda_{1}}^{-1} \mathcal{A}_{\lambda_{1}}, q_{\lambda_{1}}^{-1} \mathcal{B}_{\lambda_{1}}\right)$ together with the restriction $\left(\boldsymbol{p}^{\mathbf{1}}, \boldsymbol{q}^{\mathbf{1}}, \boldsymbol{f}^{\mathbf{1}}\right)_{*}$ of $\left(\boldsymbol{p}, \boldsymbol{q}^{\mathbf{0}}, \boldsymbol{f}\right)_{*}$ to $\Lambda_{1}=\left\{\lambda \in \Lambda_{0}: \lambda \geq \lambda_{1}\right\}$ form a pointed ANR-enriched pull-back expansion for $f$ and $(\mathcal{A}, \mathcal{B})$ (see the proof of Lemma 27 of $[5]$ ). Since $(\mathcal{A}, \mathcal{B})$ is an indecomposable overlay pair for $f$, Theorem 2 implies that $X$ is connected. So, $f$ is an $s$-sheeted covering mapping with a connected total space. Moreover, $\Psi_{*}(f)=[\mathcal{A}, \mathcal{B}]_{*}$ and $\bar{\Phi}_{*}\left([\mathcal{A}, \mathcal{B}]_{*}\right)=\mathbf{H}_{*}=\left\{H_{\lambda}, q_{\lambda \lambda^{\prime} \#}, \Lambda_{1}\right\}$, where $H_{\lambda}=f_{\lambda \#}\left(\pi_{1}\left(X_{\lambda}, *\right)\right)$, and therefore, $F_{*}\left([f]_{*}\right)=\mathbf{H}_{*}$.

Remark 1. If $Y$ is a compact Hausdorff space, one can take for $\boldsymbol{q}: Y \rightarrow \boldsymbol{Y}$ any inverse limit consisting of compact connected ANRs.

The unpointed version of Theorem 6 is the folowing.

Theorem 7. Let $Y$ be a connected paracompact space, let $f: X \rightarrow Y$ be a mapping and let $s \in \mathbb{N}$. Let $\boldsymbol{q}=\left(q_{\lambda}, \lambda \in \Lambda\right): Y \rightarrow \boldsymbol{Y}$ be an arbitrary resolution of $Y$ consisting of connected ANRs. The mapping $f$ is an s-sheeted covering mapping with connected total space if and only if there exists a mapping $f=\left(f_{\lambda}, \lambda \in \Lambda_{0}\right): \boldsymbol{X} \rightarrow \boldsymbol{Y}^{\mathbf{0}}$ over some subset $\Lambda_{0}=\left\{\lambda \in \Lambda: \lambda \geq \lambda_{0}\right\}$, such that each mapping $f_{\lambda}: X_{\lambda} \rightarrow Y_{\lambda}$ is an $s$ sheeted covering mapping with a connected total space, for every $\lambda \leq \lambda^{\prime}$, mappings $f_{\lambda}$, $f_{\lambda^{\prime}}, p_{\lambda \lambda^{\prime}}$ and $q_{\lambda \lambda^{\prime}}$ form pull-back diagrams $D_{\lambda \lambda^{\prime}}$ and $f=\lim f$. In that case $F([f])$ is the conjugacy class of $\mathbf{H}_{*}$, where $\mathbf{H}_{*}$ is obtained by $\boldsymbol{f}_{*}$ for an arbitrary points $* \in Y$ and $* \in f^{-1}(*)$.

\section{Finite-sheeted covering mappings of solenoids}

In this section we apply the developed theory to the problem of classifying all finitesheeted covering mappings over solenoids.

Let $\boldsymbol{R}=\left(r_{1}, r_{2}, \ldots\right)$ be a sequence of prime numbers, let $S_{i}, i \in \mathbb{N}$, be the circle $S^{1}$ considered as the set of all complex numbers $z$ with $|z|=1$ and let $q_{i, i+1}: S_{i+1} \rightarrow S_{i}$ be the map defined by $q_{i, i+1}(z)=z^{r_{i}}, i \in \mathbb{N}$. Let us denote by $\boldsymbol{S}$ the inverse sequence $\left(S_{i}, q_{i, i+1}\right)$. The inverse limit $\Sigma(\boldsymbol{R})=\lim \boldsymbol{S}$ is the solenoid generated by the sequence $\boldsymbol{R}=\left(r_{1}, r_{2}, \ldots\right)$. In particular, if each $r_{i}=2, \Sigma(\boldsymbol{R})$ is the dyadic solenoid. $\Sigma(\boldsymbol{R})$ is a connected, compact, metric space. Since $S^{1}$ is a compact ANR, $\boldsymbol{q}=\left(q_{i}: \Sigma(\boldsymbol{R}) \rightarrow S_{i}\right)$ : $\Sigma(\boldsymbol{R}) \rightarrow \boldsymbol{S}=\left(S_{i}, q_{i, i+1}\right)$ is also an ANR-resolution of $\Sigma(\boldsymbol{R})$, so we can use it in our considerations concerning coverings of $\Sigma(\boldsymbol{R})$. Let us now note that the fundamental progroup $\underline{\pi}_{1}(\Sigma(\boldsymbol{R}), *)$ of $\Sigma(\boldsymbol{R})$ is the inverse sequence $\mathbb{Z} \stackrel{r_{1}}{\leftarrow} \stackrel{\mathbb{Z}_{2}}{\leftarrow} \mathbb{Z} \stackrel{r_{3}}{\leftarrow} \cdots$, where $r_{i}: \mathbb{Z} \rightarrow \mathbb{Z}$ denotes multiplication of integers by $r_{i}$. Since this progroup is abelian, we do not need to distinguish the pointed and the unpointed covering mappings of $\Sigma(\boldsymbol{R})$.

Theorem 8. Let $\Sigma(\boldsymbol{R})$ be the solenoid generated by a sequence $\boldsymbol{R}=\left(r_{1}, r_{2}, \ldots\right)$ and 
let $s \in \mathbb{N} . \Sigma(\boldsymbol{R})$ admits an s-sheeted covering mapping with connected total space if and only if $s$ is not divisible by primes which occur infinitely often in the sequence $\boldsymbol{R}=\left(r_{1}, r_{2}, \ldots\right)$. Furthermore, if $\Sigma(\boldsymbol{R})$ admits an s-sheeted covering mapping with connected total space, its equivalence class is unique and the total space is homeomorphic to $\Sigma(\boldsymbol{R})$.

Proof. Let us first examine the existence of subprogroups of index $s$ of the fundamental progroup $\underline{\pi}_{1}(\Sigma(\boldsymbol{R}), *)$. $\mathbb{Z}$ has only one subgroup of index $s$ and that is $s \mathbb{Z}$. Since $\mathbb{Z} / s \mathbb{Z}=\mathbb{Z}_{s}$, it is clear that the induced mapping $r_{i}: \mathbb{Z}_{s} \rightarrow \mathbb{Z}_{s}$ is a bijection iff $r_{i}$ and $s$ are relatively prime (or equivalently if $s$ is not divisible by $r_{i}$, since $r_{i}$ is prime). Consequently, $\underline{\pi}_{1}(\Sigma(\boldsymbol{R}), *)$ has only one subprogroup of index $s$ iff $s$ is not divisible by any of the primes that occur infinitely often in the sequence $\boldsymbol{R}=\left(r_{1}, r_{2}, \ldots\right)$. According to Theorem 4, it follows that $\Sigma(\boldsymbol{R})$ admits only one equivalence class of an $s$-sheeted covering mapping $f: X \rightarrow \Sigma(\boldsymbol{R})$ with a connected total space iff $s$ is not divisible by any of the primes that occur infinitely often in the sequence $\boldsymbol{R}=\left(r_{1}, r_{2}, \ldots\right)$. Consequently, if $s$ is not divisible by any of the primes that occur infinitely often in the sequence $\boldsymbol{R}=\left(r_{1}, r_{2}, \ldots\right), \Sigma(\boldsymbol{R})$ admits a unique (up to equivalence) covering mapping with a connected total space. Conversely, if $\Sigma(\boldsymbol{R})$ admits an $s$-sheeted covering mapping with a connected total space, $s$ is not divisible by any of the primes that occur infinitely often in the sequence $\boldsymbol{R}=\left(r_{1}, r_{2}, \ldots\right)$.

Let us now assume that $f: X \rightarrow \Sigma(\boldsymbol{R})$ is an $s$-sheeted covering mapping with a connected total space $X$. Let us consider the following mapping $f=$ $\left\{f_{i}: S^{1} \rightarrow S^{1} \mid f_{i}(z)=z^{s}, i \in \mathbb{N}\right\}: S \rightarrow S$ of inverse sequences:

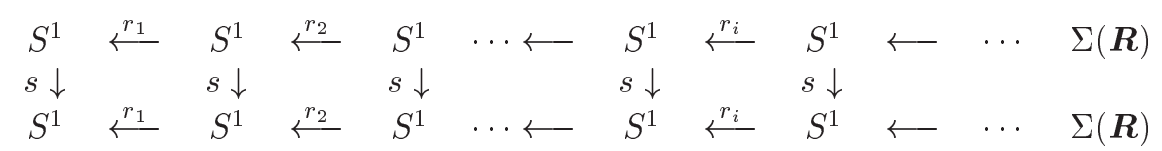

Let us denote by $f^{\prime}: \Sigma(\boldsymbol{R}) \rightarrow \Sigma(\boldsymbol{R})$ the limit of $\boldsymbol{f}$. Since $s$ is not divisible by any of the primes that occur infinitely often in the sequence $\boldsymbol{R}=\left(r_{1}, r_{2}, \ldots\right)$, there exists an index $i_{0} \in \mathbb{N}$ such that $r_{i}: \mathbb{Z}_{s} \rightarrow \mathbb{Z}_{s}$ is a bijection for each $i \geq i_{0}$, and therefore, each commutative diagram

$$
\begin{array}{rll}
S_{i} & \stackrel{z^{r_{i}}}{\longleftarrow} & S_{i+1} \\
z^{s} \downarrow & & \downarrow z^{s} \\
S_{i} & \overleftarrow{z^{r_{i}}} & S_{i+1}
\end{array}
$$

is a a pull-back diagram, $i \geq i_{0}$ (Lemma 10 of [5]). Then Theorem 7 implies that $f^{\prime}: \Sigma(\boldsymbol{R}) \rightarrow \Sigma(\boldsymbol{R})$ is an $s$-sheeted self covering of $\Sigma(\boldsymbol{R})$. Since $\Sigma(\boldsymbol{R})$ has a unique (up to equivalence) $s$-sheeted covering mapping with a connected total space, we conclude that $X$ must be homeomorphic to $\Sigma(\boldsymbol{R})$.

Remark 2. (i) The dyadic solenoid $\Sigma(2,2, \ldots)$ is a self-cover for each odd $s \in \mathbb{N}$.

(ii) Let all prime numbers occur in the sequence $\boldsymbol{R}=\left(r_{1}, r_{2}, \ldots\right)$. If each prime number occurs only finitely many times in the sequence $\boldsymbol{R}=\left(r_{1}, r_{2}, \ldots\right)$, then the 
solenoid $\Sigma(\boldsymbol{R})$ is a self-cover for each $s \in \mathbb{N}$. If each prime number occur infinitely often in the sequence $\boldsymbol{R}=\left(r_{1}, r_{2}, \ldots\right)$, then the solenoid $\Sigma(\boldsymbol{R})$ has no finite-sheeted covering mappings with a connnected total space.

Let us now consider a more general situation. Let $\left(Y_{\lambda}, q_{\lambda \lambda^{\prime}}, \Lambda\right)$ be an inverse system, where each $Y_{\lambda}=S^{1}$ and each bonding mapping $q_{\lambda \lambda^{\prime}}: Y_{\lambda^{\prime}} \rightarrow Y_{\lambda}, \lambda^{\prime} \geq \lambda$, is an $r_{\lambda \lambda^{\prime}}$-sheeted covering of $S^{1}, r_{\lambda \lambda^{\prime}} \in \mathbb{N}$. This means that $q_{\lambda \lambda^{\prime}}(z)=z^{r_{\lambda \lambda^{\prime}}}$ for each $z \in S^{1}$. Let us denote by $\boldsymbol{T}$ the set $\left\{r_{\lambda \lambda^{\prime}}: \lambda^{\prime} \geq \lambda\right\}$. We say that $n \in \mathbb{N}$ appears cofinally often in $\boldsymbol{T}$ provided for each $\left(\lambda, \lambda^{\prime}\right) \in \Lambda \times \Lambda, \lambda^{\prime} \geq \lambda$, there exists $\left(\lambda_{0}, \lambda_{1}\right) \in \Lambda \times \Lambda, \lambda_{1} \geq$ $\lambda_{0} \geq \lambda^{\prime}$, and $r_{\lambda_{0} \lambda_{1}}=n$. Let $\Sigma(\boldsymbol{T})$ be the inverse limit of the system $\left(Y_{\lambda}, q_{\lambda \lambda^{\prime}}, \Lambda\right) . \Sigma(\boldsymbol{T})$ is the solenoid generated by the set $\boldsymbol{T} . \Sigma(\boldsymbol{T})$ is a connected compact Hausdorff space and $\boldsymbol{q}=\left(q_{\lambda}: \Sigma(\boldsymbol{T}) \rightarrow Y_{\lambda}\right): \Sigma(\boldsymbol{T}) \rightarrow\left(Y_{\lambda}, q_{\lambda \lambda^{\prime}}, \Lambda\right)$ is an ANR resolution of $\Sigma(\boldsymbol{T})$. It is clear that the fundamental progroup $\underline{\pi}_{1}(\Sigma(\boldsymbol{T}), *)$ of $\Sigma(\boldsymbol{T})$ is the progroup $\left(\mathbb{Z}, r_{\lambda \lambda^{\prime}}, \Lambda\right)$, where $r_{\lambda \lambda^{\prime}}: \mathbb{Z} \rightarrow \mathbb{Z}$ denotes multiplication of integers by $r_{\lambda \lambda^{\prime}}$. Arguing as above we conclude that $\Sigma(\boldsymbol{T})$ admits an $s$-sheeted covering mapping with connected total space if and only if $s$ is relatively prime with each $r_{\lambda \lambda^{\prime}}$ which appears cofinally often in the set $\boldsymbol{T}$. Furthermore, if $\Sigma(\boldsymbol{T})$ admits an $s$-sheeted covering mapping with connected total space, its equivalence class is unique and the total space is homeomorphic to $\Sigma(\boldsymbol{T})$.

Following Timm, we say that a connected compact Hausdorff space $Y$ is $h$ - connected if $Y$ satisfies the following property: Whenever $f: X \rightarrow Y$ is a finite sheeted covering with a connected total space $X$, it follows that $X$ is homeomorphic to $Y$. It is clear that $S^{1}$ is $h$-connected. Recently, M. Timm classified $h$-connected regular 2-complexes (see [9]). Now, we can state the following corollary, which answers a question raised by Timm.

Corollary 1. Every solenoid $\Sigma(\boldsymbol{T})$ generated by a set $\boldsymbol{T}$ is $h$-connected.

\section{References}

[1] E. Bredon, Topology and Geometry, Springer, New York, 1993.

[2] R.H. Fox, On shape, Fund. Math. 74 (1972), 47-71.

[3] R.H. Fox, Shape theory and covering spaces, Lecture Notes in Math. 375, Springer, Berlin, 1974, pp. 77-90.

[4] S. Mardešić, Inverse limits and resolutions, in Shape theory and geometric topology, Proc. Conference, Dubrovnik 1981, Lecture Notes in Math. 870, Springer Verlag, Berlin, 1981, $240-253$.

[5] S. Mardešić and V. Matijević, Classifying overlay structures of topological spaces, Topology and Appl. 113 (2001), 167-209.

[6] S. Mardešić and J. Segal, Shape theory. The inverse system approach, North-Holland, Amsterdam, 1982.

[7] T.T. Moore, On Fox's theory of overlays, Fund. Math. 99 (1978), 205-211. 
[8] P. Mrozik, Images and exactness in the category of pro-groups and the lifting problem for covering projections, Glasnik. Mat. 28 (1993), 209-226.

[9] M. Timm, A classification of h-connected 2-complexes, Topology and Appl. 111(2001), 355-363.

[10] A. Zabrodsky, Covering spaces of paracompact spaces, Pacific J. Math. 14 (1964), 14891503. 